\title{
(2) OPEN ACCESS \\ Descriptive exploration of overdose codes in hospital and emergency department discharge data to inform development of drug overdose morbidity surveillance indicator definitions in ICD-10-CM
}

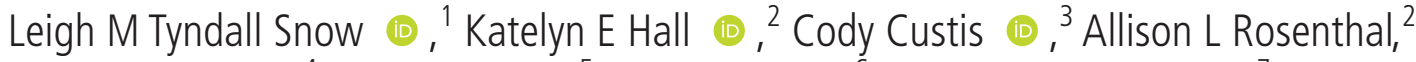 \\ Emilia Pasalic (10, ${ }^{4}$ Sarah Nechuta, ${ }^{5}$ James W Davis, ${ }^{6}$ Bretta Jane Jacquemin ${ }^{7}$ \\ Sherani R Jagroep, ${ }^{8}$ Peter Rock, ${ }^{9}$ Elyse Contreras, ${ }^{2}$ Barbara A Gabella 이, ${ }^{2}$ \\ Katherine A James ${ }^{10}$
}

- Additional material is published online only. To view please visit the journal online (http://dx.doi.org/10.1136/ injuryprev-2019-043520).

For numbered affiliations see end of article.

\section{Correspondence to} Leigh M Tyndall Snow, Analytics and Data, Blue Cross and Blue Shield of Louisiana, Baton Rouge, LA 70809, USA; tyndallsnowl@gmail.com

Received 28 February 2020 Revised 2 November 2020 Accepted 13 November 2020

Check for updates

(C) Author(s) (or their employer(s)) 2021. Re-use permitted under CC BY-NC. No commercial re-use. See rights and permissions. Published by BMJ.

To cite: Tyndall Snow LM, Hall KE, Custis C, et al.

Inj Prev 2021:27:i27-i34.

\section{ABSTRACT}

Background In October 2015, discharge data coding in the USA shifted to the International Classification of Diseases, 10th Revision, Clinical Modification (ICD-10CM), necessitating new indicator definitions for drug overdose morbidity. Amid the drug overdose crisis, characterising discharge records that have ICD-10CM drug overdose codes can inform the development of standardised drug overdose morbidity indicator definitions for epidemiological surveillance.

Methods Eight states submitted aggregated data involving hospital and emergency department (ED) discharge records with ICD-10-CM codes starting with T36-T50, for visits occurring from October 2015 to December 2016. Frequencies were calculated for (1) the position within the diagnosis billing fields where the drug overdose code occurred; (2) primary diagnosis code grouped by ICD-10-CM chapter; (3) encounter types; and (4) intents, underdosing and adverse effects.

Results Among all records with a drug overdose code, the primary diagnosis field captured $70.6 \%$ of hospitalisations (median $=69.5 \%$, range $=66.2 \%-76.8 \%$ ) and $79.9 \%$ of ED visits (median $=80.7 \%$; range $=69.8 \%-88.0 \%$ ) on average across participating states. The most frequent primary diagnosis chapters included injury and mental disorder chapters. Among visits with codes for drug overdose initial encounters, subsequent encounters and sequelae, on average $94.6 \%$ of hospitalisation records (median $=98.3 \%$; range $=68.8 \%-98.8 \%$ ) and $95.5 \%$ of ED records (median $=99.5 \%$; range $=79.2 \%-99.8 \%$ ), represented initial encounters. Among records with drug overdose of any intent, adverse effect and underdosing codes, adverse effects comprised an average of $74.9 \%$ of hospitalisation records (median $=76.3 \%$; range $=57.6 \%-81.1 \%$ ) and $50.8 \%$ of ED records ( median $=48.9 \%$; range $=42.3 \%-66.8 \%$ ), while unintentional intent comprised an average of $11.1 \%$ of hospitalisation records (median $=11.0 \%$; range $=8.3 \%-14.5 \%$ ) and $28.2 \%$ of $E D$ records ( median $=25.6 \%$; range $=20.8 \%-40.7 \%$ ).

Conclusion Results highlight considerations for adapting and standardising drug overdose indicator definitions in ICD-10-CM.

\section{INTRODUCTION Background}

The International Classification of Diseases, 10th Revision, Clinical Modification (ICD-10-CM), a coding system for all claims billing in the USA, went into effect on 1 October 2015, replacing the 9th Revision (ICD-9-CM). ${ }^{12}$ The coding transition brought with it benefits and challenges for drug overdose surveillance using hospital and emergency department (ED) discharge data. Increases in specificity of drug codes, the ability to distinguish initial encounters from subsequent encounters and sequelae, the inclusion of external cause information within drug overdose diagnosis codes and new concepts like 'underdosing' provide epidemiologists with richer data. ${ }^{1}$ However, these new features impact how injuries, including drug overdose, should be measured and interpreted using ICD-10-CM coded discharge data. ${ }^{13}$ Amid a continuing drug overdose epidemic in the USA, high-quality data and standardised surveillance methods are key for tracking and assessing drug overdose burden. ${ }^{4}$

Several studies have characterised drug overdoses using ICD-10-CM coded discharge data, but have used varying selection criteria and indicator definitions to ascertain drug overdose cases. ${ }^{5-8}$ Specifically, these indicator definitions differ on the number of diagnosis fields searched, the encounter types included, the manner of injury/intent selected and the specific ICD-10-CM codes included. Some studies searched all diagnosis fields (ie, primary diagnosis field, secondary diagnosis and all other fields available $)^{67}$ and others only included cases with a primary diagnosis of injury. ${ }^{8}$

ICD-10-CM introduced the differentiation of encounter type, with codes for initial encounter, subsequent encounter and sequelae. ${ }^{19}$ Initial encounters represent active treatment of drug overdose, while subsequent encounters represent encounters taking place 'after the patient has completed active treatment of the condition and is receiving routine care for the condition during the healing or recovery phase'. ${ }^{9}$ Sequelae are new medical conditions or complications, occurring at any point after the acute phase of the injury, which 
are residual effects of the original injury. ${ }^{9}$ Some studies have included all encounters, ${ }^{5-7}$ while others have limited inclusion to initial encounter type. ${ }^{8}$

Another difference of ICD-10-CM is that it includes manner of injury/intent within the drug overdose diagnosis code itself, while ICD-9-CM captured this information with a separate external cause of injury code. ${ }^{19}$ In addition to standard intent categories for injury (unintentional, intentional self-harm, assault and undetermined), the drug overdose codes can indicate an adverse effect or underdosing. ${ }^{9}$ Adverse effects are pharmacological effects from medication correctly prescribed and properly administered, while underdosing is the effect of using less of a drug than was prescribed. ${ }^{9}$ Another change introduced is that guidance instructs coders to choose unintentional intent if no other intent is explicitly noted in the medical record, whereas ICD-9-CM instructs coders to default to undetermined intent. ${ }^{19}$ These changes in guidance could result in systematic changes of drug overdose intent categorisation that will affect counts when stratifying drug overdoses by intent. In two studies, by Heslin et $a l$ and Moore and Barrett, intentional self-harm and assault cases were excluded in the drug overdose definitions. However, in other studies, Slavova et al and CDC researchers included these cases. $^{5-8}$

ICD-10-CM codes T36-T50 (poisoning by, adverse effect of, and underdosing of drugs, medicaments and biological substances) have been used frequently to study and surveil acute drug overdose. ${ }^{6-8} 10-13$ However, several codes from outside of the injury chapter ('Injury, poisoning and certain other consequences of external causes') could be relevant for drug overdose surveillance when coded as co-occurring conditions on the medical record. One example is O9A.2, 'Injury, poisoning and certain other consequences of external causes complicating pregnancy, childbirth and the puerperium'. In the event that someone experiences a drug overdose during pregnancy, ICD-10-CM mandates placement of O9A.2 as the primary diagnosis. ${ }^{9}$ Another example is codes for substance use with intoxication from the mental health chapter of ICD-10-CM, such as F11.22, 'Opioid dependence with intoxication', which has been used in syndrome definitions for rapidly identifying suspected drug overdose cases. ${ }^{14}$

The varying indicator definitions and selection criteria across studies reflect a lack of standardised guidance for drug overdose surveillance and variations in the structure of discharge datasets. The findings presented in this paper supported the development of several standardised guidance documents for drug overdose morbidity surveillance methods: the CSTE ICD-10-CM Injury Surveillance Toolkit, the CSTE Nonfatal Opioid Overdose Standardized Surveillance Case Definition position statement, and Drug Overdose Surveillance and Epidemiology system indicator guidance for CDC's Overdose Data to Action cooperative agreements. ${ }^{11} 1516$

This study provides a descriptive exploration of the new features in ICD-10-CM drug overdose codes and how variations in indicator definitions would affect drug overdose counts when using discharge data for drug overdose surveillance. These analyses answered the following questions:

1. How many diagnosis fields should be searched for drug overdose indicators?

2. Should drug-related ICD-10-CM codes from outside of the injury chapter be included?

3. Which encounter types should be included?

4. Which manners of injury/intents should be included?

\section{METHODS}

\section{Data}

This study employs a cross-sectional descriptive analysis of state-wide hospital and ED discharge datasets from New Jersey, Montana, New Mexico, Tennessee, Louisiana, North Carolina, Kentucky and Colorado. ${ }^{17-27}$ Hospital discharge databases use the standard uniform billing form (UB-04), created by the National Uniform Billing Committee, to standardise billing data for reimbursement. ICD-10-CM codes are assigned for reimbursement by certified, trained medical coders. Data were representative of all patient records from acute care facilities and excluded records from federal, nonacute care and psychiatric facilities. Records included all ages, sexes and admission types. Records from non-state residents were excluded. ${ }^{28}$ The nature of this non-research, secondary data analysis of de-identified data precluded patient and public involvement.

Table 1 describes the characteristics of participating states and their data.

\section{Data preparation and analyses}

Hospitalisation and ED datasets were analysed separately. To avoid duplication, patients who presented to the ED and were subsequently hospitalised were excluded from the ED datasets. These analyses used a provisional ICD-10-CM definition for drug overdose proposed by a workgroup of the national Council of State and Territorial Epidemiologists and the National Center for Injury Prevention and Control which included a diagnosis code in any field starting with T36-T50, with unintentional, intentional harm, assault or undetermined intent, and initial, subsequent or missing encounter type, (hereafter 'drug overdose codes'). The proposed definition for drug overdose excluded adverse effects and underdosing, and sequelae. Intents, including adverse effects and underdosing

\begin{tabular}{|c|c|c|c|}
\hline & \multicolumn{3}{|c|}{ Hospitalisation data } \\
\hline & $\begin{array}{l}\text { Contributed } \\
\text { data }\end{array}$ & Time span & Diagnosis code fields \\
\hline State 1 & Yes & 1 Oct 2015-31 Dec 2016 & 30 \\
\hline State 2 & Yes & 1 Oct 2015-31 Dec 2016 & $25+3 \mathrm{ECl}$ fields* \\
\hline State 3 & Yes & 1 Jan 2016-31 Dec 2016 & $18+3 \mathrm{ECl}$ fields \\
\hline State 4 & Yes & 1 Oct 2015-31 Dec 2016 & $31+5 \mathrm{ECl}$ fields \\
\hline State 5 & Yes & 1 Oct 2015-31 Dec 2016 & $18+3 \mathrm{ECl}$ fields \\
\hline State 6 & Yes & 1 Oct 2015-31 Dec 2016 & $9+3 \mathrm{ECl}$ fields \\
\hline State 7 & Yes & 1 Oct 2015-31 Dec 2016 & 95 \\
\hline \multirow[t]{3}{*}{ State 8} & Yes & 1 Oct 2015-31 Dec 2016 & $25+6 \mathrm{ECl}$ fields \\
\hline & \multicolumn{3}{|c|}{ Emergency department data } \\
\hline & $\begin{array}{l}\text { Contributed } \\
\text { data }\end{array}$ & Time span & Diagnosis code fields \\
\hline State 1 & Yes & 1 Jan 2016-31 Dec 2016 & 15 \\
\hline State 2 & Yes & 1 Oct 2015-30 Jun 2016 & $25+3 \mathrm{ECl}$ fields \\
\hline State 3 & Yes & 1 Jan 2016-31 Dec 2016 & $48+6 \mathrm{ECl}$ fields \\
\hline State 4 & Yes & 1 Jan 2016-31 Dec 2016 & 26 \\
\hline State 5 & Yes & 1 Oct 2015-31 Dec 2016 & $18+3 \mathrm{ECl}$ fields \\
\hline State 6 & No & NA & NA \\
\hline State 7 & No & NA & NA \\
\hline State 8 & No & NA & NA \\
\hline
\end{tabular}

${ }^{*} \mathrm{ECl}$ fields refer to dedicated external cause of injury code fields separate from other diagnosis codes.

$\mathrm{ECl}$, external cause of injury; NA, not applicable. 
were categorised using the fifth/sixth character of the ICD-10-CM code, and encounter types, including sequelae, were categorised using the seventh character.

\section{Analysis 1: diagnosis fields}

Discharge datasets contain a primary diagnosis followed by an array of secondary diagnosis fields allowing comorbid diagnoses to be sequenced together in a single record. To examine frequency of the first position (field) of the drug overdose codes, records with a drug overdose code according to the proposed definition were identified searching the primary diagnosis first, then secondary diagnosis fields, then external cause fields. The position of the first-mentioned drug overdose code in the record was identified. These code positions were categorised $(1,2-5,6-10,11-15,16+)$.

\section{Analysis 2: other chapters}

The ICD-10-CM codes are organised by major clinical conditions. To parsimoniously characterise possible broad co-occurring conditions among records with a drug overdose code in any field, the ICD-10-CM chapters of the coding manual (eg, 'A00-B99', 'C00-D99' and so on) of the primary diagnosis codes were examined. The primary diagnosis codes were categorised into ICD-10-CM coding chapters and quantified.

\section{Analysis 3: encounter type}

To examine the distribution of encounter types among drug overdose codes and their sequelae, records with a drug overdose code or sequelae to a drug overdose code were identified by searching all diagnosis fields, and the encounter type was categorised into initial (A), subsequent (D), sequela (S) or missing encounters based on the seventh character of the code.

\section{Analysis 4: intent}

To examine the distribution of intents among drug overdose, adverse effect and underdosing codes, records with a drug overdose, adverse effect or underdosing code were identified by searching all diagnosis fields, and the intent for the drug overdose code was categorised into unintentional, intentional self-harm, assault, undetermined, adverse effect, underdosing or missing based on the fifth/sixth character of the code. All categories of intent were analysed for completeness, though adverse effect and underdose are by definition not considered drug overdoses in this manuscript.

State staff tabulated frequencies and percentages for code position categories, encounter types, intents and primary diagnosis chapters using SAS V.9.4. ${ }^{29}$ State-specific results and averages of all state results are presented.

\section{RESULTS}

Hospitalisations from 1 October 2015 through 31 December 2016 with drug overdose codes in any field ranged from 976 records per state to 9565 records per state across eight states. ED visits with drug overdose codes in any field ranged from 9086 records per state to 36326 records per state across five states during the time periods examined in each state.

\section{Analysis 1: diagnosis fields}

The distribution of the field-position of the first-mentioned drug overdose code in each record for hospitalisation (A) and ED visit (B) data are shown in figure 1 and online supplemental table 1. Among hospitalisations with a drug overdose code, the primary diagnosis field captured $70.6 \%$ of hospitalisations (median $=69.5 \%$; range $=66.2 \%-76.8 \%$ ) on average across participating states. Among ED visits with a drug overdose code, the primary diagnosis field captured $79.9 \%$ (median $=80.7 \%$; range $=69.8 \%-88.0 \%)$ on average across participating states. The first 10 fields captured $94.3 \%$ of hospitalisations $($ median $=94.1$; range $=90.8 \%-100.0 \%)$ and $99.2 \%$ of $\mathrm{ED}$ visits (median $=99.7 \%$; range $=97.2 \%-99.9 \%$ ).

\section{Analysis 2: other chapters}

Figure 2 and online supplemental table 2 show the most common chapters of ICD-10-CM codes that were in the primary diagnosis field when the drug overdose code was in any field position.

Among hospitalisation records with a drug overdose code in any field position, on average, $72.5 \%$ (median $=71.1 \%$; range $=68.1 \%-79.2 \%$ ) of the primary diagnosis fields included codes from the 'S00-T88: Injury, Poisoning, and Other

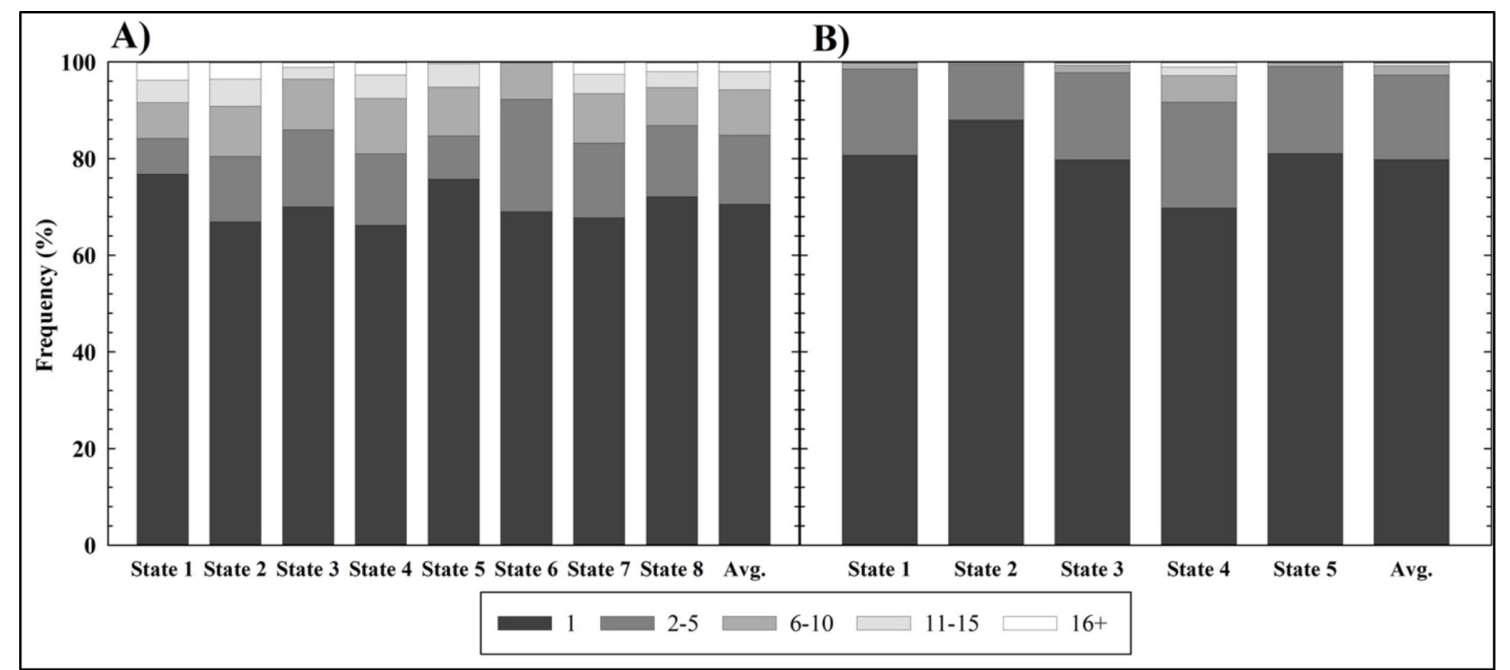

Figure 1 Analysis 1: distribution of the field position of the first-mentioned drug overdose code* in each claim record. (A) Discharge data from eight states' hospitalisation databases. (B). Discharge data from five states' ED visit databases. *Drug overdose diagnoses include ICD-10-CM codes starting with T36-T50 including unintentional, intentional harm, assault or undetermined intents, and initial, subsequent or missing encounter type. Avg., average; ED, emergency department; ICD-10-CM, International Classification of Diseases, 10th Revision, Clinical Modification. 


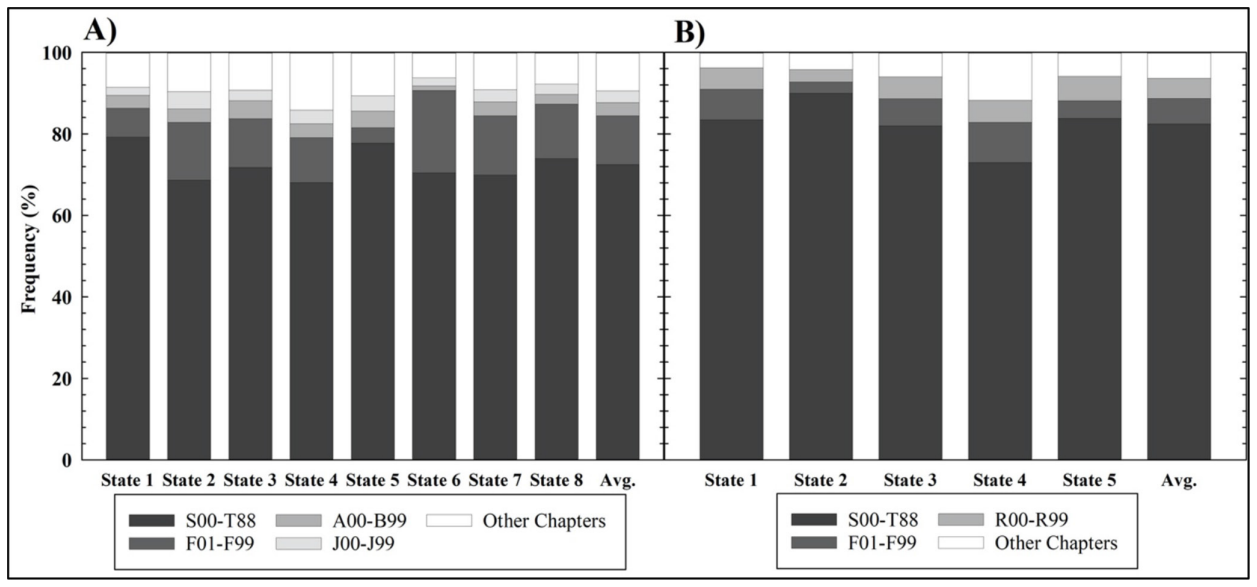

Figure 2 Analysis 2: top chapters of primary diagnosis for records with a drug overdose diagnosis code* in any field. (A) Discharge data from eight states' hospitalisation databases. Top four most common chapters are displayed. (B) Discharge data from five states' ED visit databases. Top three most common chapters are displayed. *Drug overdose diagnoses include ICD-10-CM code beginning with T36-T50 including unintentional, intentional harm, assault or undetermined intents, and initial, subsequent or missing encounter type. Avg., average; ED, emergency department; ICD10-CM, International Classification of Diseases, 10th Revision, Clinical Modification.

Consequences of External Causes' chapter. The second most common chapter of primary diagnosis for all but one state was 'F01-F99: Mental, Behavioral, and Neurodevelopmental Disorders' chapter with an average of $12.0 \%$ (median $=12.7 \%$; range $=3.8 \%-20.2 \%)$. State 5 's second most common chapter was 'A00-B99: Certain Infectious and Parasitic Diseases' (4.1\%). The third most common chapter varied: 'A00-B99' for states 1, 3 and 7; 'J00-J99: Diseases of the Respiratory System' for states 2, 6 and 8; 'F01-F99' for state 5 and 'I00I99: Diseases of the Circulatory System' for state 4. All other chapters represented an average of $12.1 \%$ of primary diagnosis codes (median $=12.0 \%$; range $=7.4 \%-17.1 \%$ ).

Among ED visit records with a drug overdose code in any field position, on average, $82.4 \%$ (median $=83.5 \%$; range $=73.0 \%-$ $89.9 \%$ ) of the primary diagnosis fields included codes from the 'S00-T88' chapter. The second most common chapter varied. States 1, 3 and 4 had 'F01-F99' as their second most common chapter, with an average of $6.3 \%$ (median $=6.7 \%$; range $=2.8 \%-9.9 \%$ ). States 2 and 5 had 'R00-R99: Symptoms, Signs and Abnormal Clinical and Laboratory Findings, Not Elsewhere Classified' as their second most common chapter, with a participating state average of $5.0 \%$ (median $=5.3 \%$; range $=3.0 \%-5.9 \%)$. The third most common chapter was 'R00-R99' for states 1, 3 and 4, and 'F01-F99' for states 2 and 5. All other chapters represented an average of $6.3 \%$ primary diagnosis codes $($ median $=5.9 \%$; range $=3.8 \%-11.8 \%)$.

\section{Analysis 3: encounter type}

Figure 3 and online supplemental table 3 show the frequency of encounter types captured among drug overdose records, subsequent encounters and sequelae. Among hospitalisations, on average, initial encounters represented $94.6 \%$ $($ median $=98.3 \% ; \quad$ range $=68.8 \%-98.8 \%) . \quad$ Subsequent

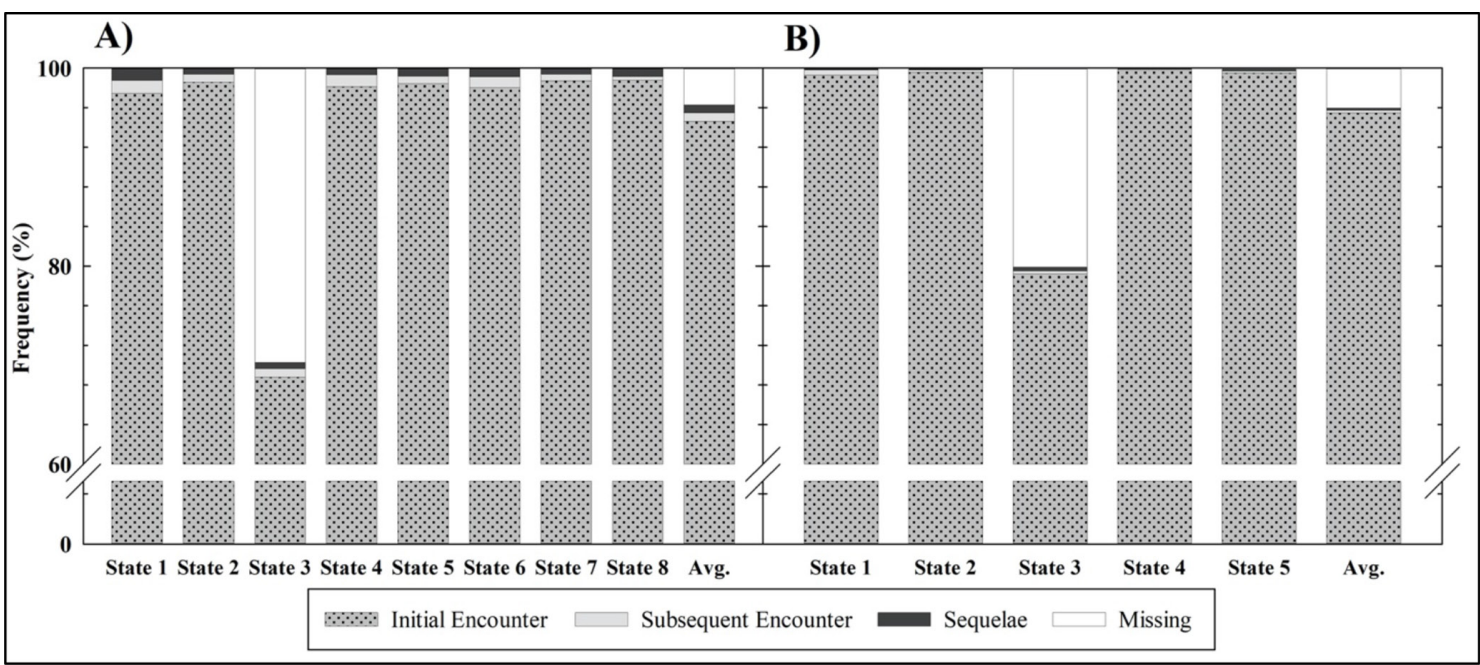

Figure 3 Analysis 3: frequency of encounter type among records with an overdose diagnosis code* or sequela in any field. (A) Discharge data from eight states' hospitalisation databases. (B) Discharge data from five states' ED visit databases. *Drug overdose diagnosis codes include ICD-10-CM codes starting with T36-T50 including unintentional, intentional harm, assault or undetermined intents, and initial, subsequent or missing encounter type. This analysis included records with overdose codes and codes for sequelae to overdose. Avg., average; ED, emergency department; ICD-10-CM, International Classification of Diseases, 10th Revision, Clinical Modification. 


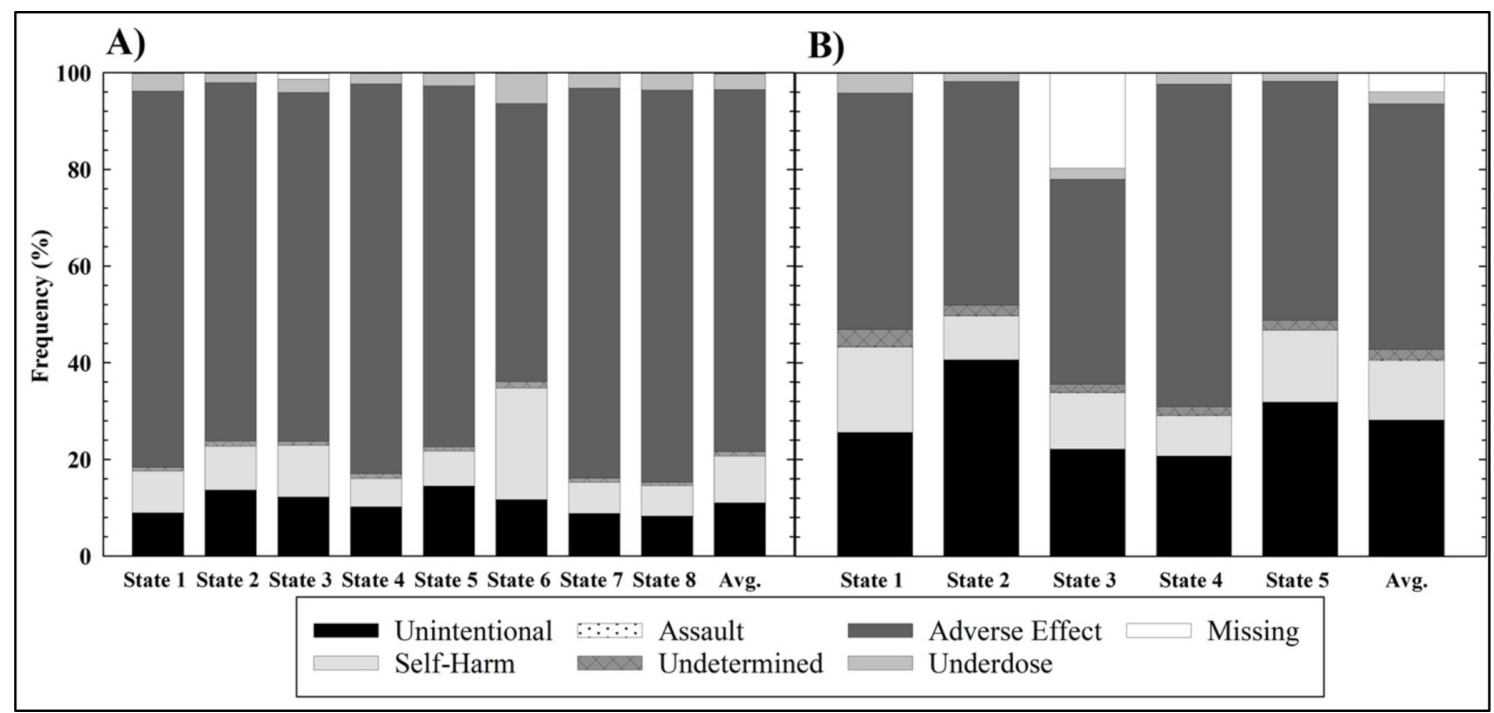

Figure 4 Analysis 4: frequency of intent among records with a drug overdose diagnosis code* in any field. (A) Discharge data from eight states' hospitalisation databases. (B) Discharge data from five states' ED visit databases. Assault category is too small to view. *Drug overdose diagnosis codes include ICD-10-CM codes starting with T36-T50 including unintentional, intentional harm, assault or undetermined intents, and initial, subsequent or missing encounter type. This analysis included overdose records with overdose codes of any intent, adverse effects codes and underdosing codes. Avg., average; ED, emergency department; ICD-10-CM, International Classification of Diseases, 10th Revision, Clinical Modification.

encounters made up an average of $0.9 \%$ (median $=0.8 \%$; range $=0.4 \%-1.3 \%)$ and sequelae $0.8 \% \quad$ (median $=0.7 \%$; range $=0.6 \%-1.2 \%)$ of hospitalisations. In state 3 , encounter type was missing for $29.7 \%$ of hospitalisation records.

Among ED records, on average, initial encounters were $95.5 \%$ (median $=99.5 \%$; range $=79.2 \%-99.8 \%)$. Subsequent encounters were $0.3 \%$ (median $=0.2 \%$; range $=0.1 \%-0.5 \%$ ) and sequelae were $0.2 \%$ (median $=0.2 \%$; range $=0.1 \%-0.4 \%$ ) of $\mathrm{ED}$ records. In state 3, encounter type was missing for $20.0 \%$ of ED records.

\section{Analysis 4: intent}

The frequency of the intents captured among drug overdose, adverse effect, and underdosing records is shown in figure 4 and online supplemental table 4 . Among hospitalisations, adverse effects codes were present across participating states in an average of $74.9 \%$ of records with drug overdose codes (median $=76.3 \%$; range $=57.6 \%-81.1 \%$ ). Among ED visits, adverse effects codes were present across participating states in an average of $50.8 \%$ of records with drug overdose codes (median $=48.9 \%$; range $=42.3 \%-66.8 \%$ ). Unintentional intent represented an average of $11.1 \%$ of hospitalisations $\quad($ median $=11.0 \%$; $\quad$ range $=8.3 \%-14.5 \%)$ and $28.2 \%$ of ED visits (median $=25.6 \%$; range $=20.8 \%-$ $40.7 \%$ ), followed by self-harm with $9.6 \%$ of hospitalisations (median $=7.9 \% ;$ range $=5.8 \%-23.0 \%$ ) and $12.3 \%$ of ED visits (median $=11.6 \%$; range $=8.3 \%-17.6 \%$ ). On average, undetermined, assault and underdosing intents represent $4.3 \%$ (median $=3.8 \%$; range $=3.1 \%-7.7 \%$ ) of hospitalisations and $4.8 \%$ (median $=4.1 \%$; range $=3.8 \%-7.8 \%$ ) of ED visits, respectively. State 3 had a relatively large percentage of missing intents among hospitalisations and ED visits compared with other states.

\section{DISCUSSION}

The analyses highlight key features of drug overdose indicators to consider when using ICD-10-CM coded data.
First, limiting selection to records with an ICD-10-CM drug overdose code in the primary diagnosis field included, on average, only $70.6 \%$ of hospitalisations and $79.9 \%$ of ED visits with a drug overdose code in the record. This suggests that a definition that searches only the primary diagnosis field would likely undercount drug overdose cases. While in hospital/inpatient settings, the primary diagnosis, known as the 'principal' diagnosis, has been confirmed by a physician, the same is not necessarily true in the ED setting. ${ }^{9}$ Healthcare Cost and Utilization Project noted in a 2011 report that 'the ED visit often focuses on the symptom-based evaluation of differential diagnoses' going on to state that 'several conditions may have relevance to the 'reason' for the ED visit, and all-listed diagnoses may need to be considered. ${ }^{30}$ Based on their study findings, Slavova et al suggested that 'heroin or other opioid poisoning surveillance definitions that include multiple diagnoses (firstlisted and secondary) would identify a high percentage of truepositive cases'. ${ }^{12}$

Given that the ordering of codes beyond the primary diagnosis is not standardised, any broader limitation in the number of fields to search (eg, limiting to 5 or 10 fields) could arbitrarily bias comparisons across states, which have differing dataset structures. For example, $100 \%$ of the drug overdose codes in hospitalisation data were captured in the first 10 fields for state 6 , a state with nine fields. In contrast, only $93.4 \%$ of records were captured in the first 10 fields for state 7 , a state with 95 fields. Among states in this study, the number of fields in the hospitalisation data ranged from 9 to 95 . At least one of the participating states store their diagnosis codes in a vertical relational database without meaningful coding order past the primary diagnosis, rather than as horizontal flat files. ${ }^{19}$ Including all available fields could minimise bias when comparing drug overdose rates across states and prevent underestimation of drug overdoses.

Second, codes from outside the injury chapter are somewhat common in the primary diagnosis field, with codes T36-T50 as secondary codes. Among the drug overdose records examined, 
the primary diagnosis codes most often came from either the injury chapter or the mental health chapter. Other codes of interest, including O9A.2 (Injury or poisoning complicating pregnancy), were relatively rare, appearing as the primary diagnosis in less than $0.5 \%$ of drug overdose records across all states (see online supplemental table 2). The primary diagnosis of mental health conditions with subsequent drug overdose codes may represent co-occurring mental health and substance use disorders, or substance use with intoxication codes. Additionally, certain infectious diseases could reflect abscesses from injection drug use or unsafe sex, respiratory disease might reflect a pre-existing condition worsened by chronic smoking of a drug, and circulatory problems could be a complication of chronic drug use, particularly in the case of stimulant use. Further research, including medical record review, could characterise records with both drug overdose and substance use disorder and intoxication codes and the physician documentation that supports each. This could reveal how incorporating codes for co-occurring conditions might affect the sensitivity or specificity of indicator definitions that rely on primary diagnosis codes only. However, expanding selection criteria to all diagnosis fields could capture drug overdose cases with relevant mental disorders, organ failure or injuries during pregnancy coded as the primary diagnosis.

Third, subsequent encounters and sequelae represent a small proportion of ICD-10-CM codes in the T36-T50 range. Given that subsequent and sequela encounters do not represent new drug overdoses, their inclusion could potentially count a single drug overdose multiple times, modestly inflating measures of drug overdose incidence.

Fourth, the distribution of cases across intent categories varied across states. The change in coding guidelines that instructs coders to default to accidental intent when the intent of the injury is unspecified is likely to impact the number of cases within each intent category in ICD-10-CM coded data. Future research exploring how medical coders assign intent categories is warranted.

Adverse effects constitute a relatively large proportion of records with codes T36-T50, while codes for underdosing are relatively rare. Adverse effects could represent common sideeffects which are inconsistent with a drug overdose. Including adverse effects could dramatically inflate estimates of drug overdose-related discharges. Underdosing can represent noncompliance or improper administration and is also inconsistent with a drug overdose. ${ }^{9}$ To prevent overestimation of drug overdoses, researchers should exclude both adverse effects and underdosing. ${ }^{15}$

Next steps to validate drug overdose indicators include replication of these analyses with nationally representative data, and medical chart review to assess the predictive value of codes for drug overdose, adverse effects and substance use with intoxication, and to understand how medical coders categorise drug overdose intent. Appropriate training for medical coders in applying ICD-10-CM codes for drug overdose may alleviate missing data and benefit end users.

\section{Limitations}

This study had several limitations. First, differences in patient reporting, physician documentation, drug screening availability, and hospital or state reporting policies could result in differences in medical coding accuracy between hospital systems and across states. However, discharge data from hospitals, standardised using ICD-10-CM and UB-04, are generally considered complete, comparable and reliable. ${ }^{30}$ Second, the ICD-10-CM codes were not validated through medical record review to determine the predictive value for identifying drug overdoses. Third, reporting completeness was affected by the ICD-10-CM transition, with some hospitals reporting incomplete or late data for quarter 4 2015-2016. Fourth, one state was missing the encounter type and intent portions of a large proportion of codes. Missing data were not excluded from this simple descriptive analysis, potentially distorting averages for analyses 3 and 4. Medians are presented along with averages excluding state 3's results in online supplemental table 1, for comparison. Fifth, participating states were self-selected and not representative of the USA, limiting the generalisability of the findings. There could also be heterogeneity in drug overdose patterns across states due to availability and variability of drug types, general population health and differing healthcare infrastructures, which may not be reflected in the sample of states for this project. However, these analyses were meant to inform indicator development and to test the suitability of the proposed methodologies for use in drug overdose surveillance, rather than to accurately describe characteristics of drug overdose discharges across the country. Participating epidemiologists from across the country had access to state-wide ICD-10-CM coded discharge data, informing surveillance methods development with these analyses just as these data became available.

\section{CONCLUSIONS}

Descriptive analyses of discharge data produced consistent results across states and across the two data sources which have informed the standardisation of drug overdose indicator definitions for hospital and ED discharges coded in ICD-10-CM. These standardised definitions have been shared in toolkits for epidemiologists and as guidance for CDC's funded partners. ${ }^{111516}$ Consistent instructions across all of these surveillance tools include: search all available diagnosis fields for drug overdose ICD-10-CM codes; exclude subsequent encounters and sequelae from indicators intended to approximate drug overdose incidence; exclude underdosing and adverse effects from drug overdose incidence indicators; and use codes T36T50 for drug overdose surveillance with discharge data.

\section{What is already known on the subject}

- Use of the International Classification of Diseases, 10th Revision, Clinical Modification (ICD-10-CM) became effective on 1 October 2015.

- Without national standards, researchers and epidemiologists have used varied methods and indicator definitions to measure drug overdose morbidity in hospital and emergency department discharge data coded with ICD-10-CM codes.

What this study adds

- This manuscript presents descriptive analyses of hospital and emergency department data, discussing how the analyses informed the development of standardised ICD-10-CM drug overdose surveillance indicator definitions by national organisations. 


\section{Author affiliations}

'Office of Public Health, Louisiana Department of Health, Baton Rouge, Louisiana, USA

${ }^{2}$ Colorado Department of Public Health and Environment, Denver, Colorado, USA ${ }^{3}$ Montana Department of Public Health and Human Services, Helena, Montana, USA ${ }^{4}$ National Center for Injury Prevention and Control, Centers for Disease Control and Prevention, Atlanta, Georgia, USA

${ }^{5}$ Tennessee Department of Health, Office of Informatics and Analytics, Nashville, TN, USA

${ }^{6}$ New Mexico Department of Health, Injury \& Behavioral Epidemiology Bureau, Santa $\mathrm{Fe}$, New Mexico, USA

${ }^{7}$ New Jersey Department of Health, Center for Health Statistics and Informatics, Trenton, New Jersey, USA

${ }^{8}$ North Carolina Department of Health and Human Services, Injury and Violence Prevention Branch, Raleigh, North Carolina, USA

${ }^{9}$ Kentucky Injury Prevention and Research Center, University of Kentucky, Lexington, Kentucky, USA

${ }^{10}$ Colorado School of Public Health, University of Colorado Anschutz Medical Campus, Aurora, Colorado, USA

\section{Acknowledgements Members of the Council of State and Territorial} Epidemiologists' ICD-10-CM Drug Poisoning Indicators Workgroup whose work in collaboration with the National Center for Injury Prevention and Control is reflected in this paper.

Contributors LMTS designed the analysis plan, performed data analysis, and drafted and revised the paper. KEH and CC designed the analysis plan, created standardised code, performed data analysis, and drafted and revised the paper. ALR designed the analysis plan and drafted and revised the paper. EP provided workgroup leadership, designed the analysis plan, performed data analysis, drafted and revised the paper, and provided mentorship to other authors. SN, JWD, BJJ, SRJ and PR designed the analysis plan, performed data analysis, and revised the paper. BAG provided workgroup leadership, designed the analysis plan, revised the paper, and provided mentorship to other authors. EC and KAJ revised the paper and provided mentorship to other authors.

Funding This work was supported in part by the States of Montana and New Jersey, the Atlanta-Carolinas High-Intensity Drug Trafficking Area programme (HIDTA): G19GA0010A, and the following cooperative agreements from the CDC. CO: 6NU17CE002719-02, KY: 6NU17CE924880-03-0, LA: 6NU17CE924870-03-02, NM: 5NU17CE002727-03-00, TN: 5NU17CE002731-02-00 and

5NU17CE924899-02-00, CSTE: NU380T000297-01-00.

Disclaimer The findings and conclusions in this paper are those of the authors and do not necessarily represent the official position of the CDC, Council of State and Territorial Epidemiologists, the state governments of Montana, Colorado, New Jersey, Louisiana, Kentucky, New Mexico, Tennessee or North Carolina, the Atlanta-Carolinas HIDTA, or the Drug Enforcement Administration.

Competing interests LMTS, SN, PR, JWD and BAG report grant funding from the CDC during the conduct of this study. All other authors have nothing to disclose.

Patient consent for publication Not required.

Provenance and peer review Commissioned; externally peer reviewed.

Data availability statement Data may be obtained from a third party and are not publicly available. All data represented in the figures in this manuscript are provided in the supplemental tables. The databases which provided the de-identified participant data used for the analyses are owned by eight different entities and are subject to those owners' privacy and confidentiality policies. More information on these databases can be found at the following websites: Colorado: https://cha. com/data-reporting/colorado-hospital-utilization-data/; Kentucky: https://chfs.ky. gov/agencies/ohda/Pages/hfsd.aspx; Louisiana: http://ldh.la.gov/index.cfm/page/ 2194; Montana: https://dphhs.mt.gov/publichealth/Epidemiology/OESS-MHDDS; New Jersey: https://www.ni.gov/health/healthcarequality/health-care-professionals/ njddcs/; New Mexico: https://nmhealth.org/about/erd/hsep/edd/; North Carolina: https://schs.dph.ncdhhs.gov/;Tennessee: https://www.tn.gov/health/health-programareas/statistics/special-reports/hdds.html.

Supplemental material This content has been supplied by the author(s). It has not been vetted by BMJ Publishing Group Limited (BMJ) and may not have been peer-reviewed. Any opinions or recommendations discussed are solely those of the author(s) and are not endorsed by BMJ. BMJ disclaims all liability and responsibility arising from any reliance placed on the content. Where the content includes any translated material, BMJ does not warrant the accuracy and reliability of the translations (including but not limited to local regulations, clinical guidelines, terminology, drug names and drug dosages), and is not responsible for any error and/or omissions arising from translation and adaptation or otherwise.

Open access This is an open access article distributed in accordance with the Creative Commons Attribution Non Commercial (CC BY-NC 4.0) license, which permits others to distribute, remix, adapt, build upon this work non-commercially, and license their derivative works on different terms, provided the original work is properly cited, appropriate credit is given, any changes made indicated, and the use is non-commercial. See: http://creativecommons.org/licenses/by-nc/4.0/.

\section{ORCID iDs}

Leigh M Tyndall Snow http://orcid.org/0000-0003-0929-0609

Katelyn E Hall http://orcid.org/0000-0003-2588-3009

Cody Custis http://orcid.org/0000-0002-2685-9388

Emilia Pasalic http://orcid.org/0000-0003-3692-3424

Barbara A Gabella http://orcid.org/0000-0001-6301-116X

\section{REFERENCES}

1 Injury Surveillance Workgroup 9. The transition from ICD-9-CM to ICD-10-CM: guidance for analysis and reporting of injuries by mechanism and intent. Atlanta, GA, 2016. http://c.ymcdn.com/sites/www.safestates.org/resource/resmgr/isw9//SW9_ FINAL Report.pdf

2 National Center for Health Statistics. International Classification of Diseases, (ICD10-CM/PCS) Transition - Background, 2015. Available: https://www.cdc.gov/nchs/icd/ icd10cm_pcs_background.htm [Accessed 19 Jan 2020].

3 Cartwright DJ. Icd-9-Cm to ICD-10-CM codes: what? why? how? Adv Wound Care 2013;2:588-92.

4 Scholl L, Seth P, Kariisa M, et al. Drug and Opioid-Involved overdose deaths - United States, 2013-2017. MMWR Morb Mortal Wkly Rep 2018;67.

5 Centers for Disease Control and Prevention. 2019 annual surveillance report of drug-related risks and outcomes — United States surveillance special report, 2019 Available: https://www.cdc.gov/drugoverdose/pdf/pubs/2019-cdc-drug-surveillancereport.pdf [Accessed 7 Dec 2019].

6 Heslin KC, Owens PL, Karaca Z, et al. Trends in opioid-related inpatient stays shifted after the US transitioned to ICD-10-CM diagnosis coding in 2015. Med Care 2017; 55:918-23.

7 Moore BJ, Barrett ML. Case study: exploring how Opioid-Related diagnosis codes translate from ICD-9-CM to ICD-10-CM, 2017. Available: https://www.hcup-us.ahrq. gov/datainnovations/ICD-10CaseStudyonOpioid-RelatedIPStays042417.pdf [Accessed 30 Sep 2019].

8 Slavova S, Costich JF, Luu H, et al. Interrupted time series design to evaluate the effect of the ICD-9-CM to ICD-10-CM coding transition on injury hospitalization trends. Inj Epidemiol 2018:5:36.

9 National Center for Health Statistics. ICD-10-CM Official Guidelines for Coding and Reporting FY 2020, 2019. Available: https://www.cdc.gov/nchs/data/icd/ 10cmguidelines-FY2020_final.pdf

10 Centers for Disease Control and Prevention. CDC's Opioid Overdose Indicator Support Toolkit: Guidance for Reporting on Opioid-Related mortality, morbidity, and PDMP indicators, Version 3.02018.

11 Council of State and Territorial Epidemiologists. ICD-10-CM injury surveillance methods toolkit. Available: https://resources. cste.org/Injury-Surveillance-MethodsToolkit [Accessed 6 Dec 2019].

12 Slavova S, Quesinberry D, Costich JF, et al. ICD-10-CM-Based definitions for emergency department opioid poisoning surveillance: electronic health record case confirmation study. Public Health Rep 2020;135:262-9.

13 National Center for Health Statistics. ICD-10-CM tabular list of diseases and injuries, 2019. Available: https://www.cdc.gov/nchs/icd/icd10cm.htm

14 Vivolo-Kantor AM, Seth P, Gladden RM, et al. Vital Signs: Trends in Emergency Department Visits for Suspected Opioid Overdoses - United States, July 2016-September 2017. MMWR Morb Mortal Wkly Rep 2018;67:279-85.

15 Vivolo-Kantor AM, Pasalic E, Liu S. Defining indicators for drug overdose emergency department visits and hospitalizations in ICD-10-CM coded discharge data. Inj Prev 2021:27:i49-54.

16 Council of State and Territorial Epidemiologists. Nonfatal opioid overdose standardized surveillance case definition. Atlanta, GA, 2019. https://cdn.ymaws.com/ www.cste.org/resource/resmgr/ps/2019ps/PS_Nonfatal_Opioid_Overdose_.pdf

17 Colorado Hospital Association. Hospital discharge dataset. Available: https://cha.com/ data-reporting/ [Accessed 1 Oct 2015 - 31 Dec 2016].

18 Kentucky Outpatient Services Database and Kentucky Inpatient Hospitalization Claims Files. Cabinet for health and family services, office of health data and analytics. Available: https://chfs.ky.gov/agencies/dph/Documents/datarg2018HIDOSD.pdf [Accessed 1 Oct 2015 - 31 Dec 2016]

19 Louisiana Hospital Association. Louisiana emergency department discharge data, 2016. Available: http://ldh.la.gov/index.cfm/subhome/46 [Accessed 1 Jan 2016- 31 Dec 2016].

20 Louisiana Hospital Association. Louisiana Hospital inpatient discharge database. Available: http://ldh.la.gov/index.cfm/page/2192 [Accessed 1 Oct 2015 - 31 Dec 2016].

21 Montana Hospital Discharge Data System. Data courtesy of the montana Hospital association. Available: https://dphhs.mt.gov/publichealth/Epidemiology/OESS-MHDDS [Accessed 1 Oct 2015 - 31 Dec 2016]. 


\section{Original research}

22 New Jersey hospital discharge data collection system, Trenton, NJ: new Jersey department of health, healthcare quality assessment. Available: https://www.nj.gov/health/ healthcarequality/health-care-professionals/njddcs/ [Accessed 1 Oct 2015 - 31 Dec 2016].

23 New Mexico Department of Health/Health Systems Epidemiology Program. New Mexico interim emergency department data system, 2016. Available: https:// nmhealth.org/about/erd/hsep/edd/ [Accessed 1 Jan 2016- 31 Dec 2016].

24 New Mexico Hospital Inpatient Discharge Data. New Mexico department of Health/ Health systems epidemiology program, 2016. Available: https://nmhealth.org/about/ erd/hsep/hidd/ [Accessed 1 Jan 2016- 31 Dec 2016].

25 North Carolina state center for health statistics. North Carolina hospital discharge data. Available: https://schs.dph.ncdhhs.gov/ [Accessed 1 Oct 2015 - 31 Dec 2016].

26 Tennessee Department of Health. Tennessee hospital discharge database system. Available: https://www.tn.gov/health/health-program-areas/statistics/special-reports/ hdds.html [Accessed 1 Oct 2015 - 31 Dec 2016].
27 Colorado Hospital Association. Emergency department visit dataset. Available: https:// cha.com/data-reporting/ [Accessed 1 Jan 2016- 31 Dec 2016].

28 Centers for Disease Control and Prevention. State injury indicators report: Instructions for preparing 2016 mortality data. 2016. Atlanta, GA, 2016. https://www.cdc.gov/injury/pdfs/2016_state_injury_indicator_instructions508.pdf 29 SAS. SAS 9.4

30 Senathirajah M, Owens P, Mutter RNM. HCUP methods series report \# 2011-03: special study on the meaning of the First-Listed diagnosis on emergency department and ambulatory surgery records, 2011. Available: https://www.hcup-us.ahrq.gov/ reports/methods/2011_03.pdf [Accessed 7 Dec 2019].

31 Pasalic ES, Vivolo-Kantor AM, Martinez P. Comparing syndromic data to discharge data to measure opioid overdose emergency department visits. Online J Public Health Inform 2019;11. 